\title{
Bipolar disorder and linkage to Xq28
}

Sir - X-linkage in bipolar disorder is a contested issue, with diverse findings and arguments ranging from population differences to type I and type II errors (see ref. 1 for review). In the March 1994 issue of Nature Genetics, Bocchetta et al. ${ }^{2}$ discussed X-linked inheritance in bipolar disorder, with special reference to the glucose-6phosphate dehydrogenase (G6PD) locus. They presented new data on their Sardinian population while referring to our own work on this topic ${ }^{3,4}$. We should like to make some additional points.

Most previous reports on linkage between bipolar disorder and G6PD were based on phenotypic data G6PD enzyme activity ${ }^{1}$. Bocchetta $e t$ $a .^{2}$ adopted a similar approach. However, misclassifications in assigning G6PD phenotypes are known to occur ${ }^{4-6}$. Recently we proposed that DNA polymorphisms supersede the less informative phenotypicmarker ${ }^{4}$. In fact, the G6PD Mediterranean mutation (G6PD Med ) itself can now be assayed ${ }^{7}$, and this led to a reversal of our previous linkage results ${ }^{4}$.

Bocchetta et al. ${ }^{2}$ noted differences in the prevalence of G6PD enzymatic deficiency between bipolar (males, $20.8 \%$; females, $36.4 \%$ ) and nonbipolar (males, $7.8 \%$; females, $25.2 \%$ ) patients suggesting a marker-disease association. For several reasons an alternative interpretation is in order. First, given the large number of comparisons (nine disease categories within and between sexes), the actual statistical differences are much smaller than those reported due to multiple test effects. Second, in their definition of bipolar disorder, the authors included several conditions whose relatedness to bipolar I disorder (depression and mania), the 'core phenotype' in the bipolar spectrum, is not clear-cut, especially when they occur in a general population sample (as opposed to aggregation in families). These are bipolar II disorder (depression and hypomania) and schizoaffective illness. Indeed, when bipolar I patients are examined separately, the difference in prevalences is lost (males, $10.7 \%$; females, $30.9 \%$ versus
$7.8 \%$ and $25.2 \%$, respectively).

Bocchetta et al. ${ }^{2}$ view other studies as consistent with a relationship between bipolar disorder and G6PD deficiency ${ }^{4,8,9}$. However, one of these studies ${ }^{8}$ was based on a single case, a woman with G6PD deficiency who suffered acute paranoid episode with quick resolution; the author concluded that organic delirium due to acute haemolysis (as opposed to bona fide mental disorder) was the likely diagnosis. Other, more systematic surveys of G6PD activity in psychiatric populations, did not find statistically significant differences in the prevalence of G6PD deficiency ${ }^{9-14}$. As for our previous study ${ }^{4}$, Boccetta et al. observed that 10 of the 11 affectively ill subjects in one pedigree (no.009) are G6PD deficient, suggesting a possible disease-marker association. However, all four unaffected subjects in that pedigree, whose genotypes were tested or could be deduced and who are well past the risk period for bipolar disorder, also carry the G6PD mutation. Given the pattern of transmission in the pedigree, this outcome cannot be wholly attributed to non-penetrance among the unaffecteds; in our reanalysis ${ }^{4}$, there was no evidence of linkage with either high $(95 \%)$ or low $(5 \%)$ penetrance bounds.

Recently, we studied miscrosatellite markers mapped to Xq27-28 in 11 newbipolar pedigrees obtained in the United States and Israel. Four of the five Israeli pedigrees were Middle Eastern; X-linked inheritance was thought to be more pronounced among some Middle Eastern and Mediterranean (the locale for Bocchetta et al.'s study) population ${ }^{1}$. Our ascertainment and diagnostic procedures were described in detail elsewhere ${ }^{15}$. The marker loci (probe name in parenthesis) are: $F 8$ (F81A), GABRA3 (MGD341) and DXS297 (VK23F). F8 is located close to the G6PDlocus; GABA3 is $5 \mathrm{cM}$ proximal to this cluster, and DXS297 (an Xq27 marker) is about $15 \mathrm{cM}$ proximal to $G A B R A 3^{15}$. Linkage analysis was carried out as described ${ }^{16}$, using the following parameters: X-linked dominant trait; affected phenotype, bipolar disorder or recurrent major depression; linear age-at-onset correction with $80 \%$ penetrance at high age; $1 \%$ disease allele frequency, and $0.1 \%$ penetrance for phenocopies. Both the pairwise and multipoint lod scores were negative for all families combined. Three families showed small positive lod scores $\left(Z_{\max }<1.5\right)$; the linkage heterogeneity test was not statistically significant.

In conclusion, support for linkage of bipolar disorder to $\mathrm{Xq} 28$ is diminished on re-evaluation of the evidence. But high lod scores in some of the earlier studies (whose status, however, is unclear pending followup and analysis with DNA markers), together with modest positive lod scores with DNA markers in other pedigrees $^{1}$, are sufficiently intriguing to warrant further examination of this long-standing hypothesis.

\section{Miron Baron \\ Richard E. Straub \\ Thomas Lehner \\ Jean Endicott \\ Jurg Ott \\ T. Conrad Gilliam}

New York State Psychiatric

Institute and Columbia

University College of Physicians

and Surgeons, New York,

New York 10032, USA

\section{Bernard Lerer}

Department of Psychiatry, Hadassah-Hebrew University

Medical Center,

Jerusalem 91120, Israel

R.E.S. current address: Department of Psychiatry, Box 980710, Medical College of Virginia, Richmond, Virginia 23928, USA

\section{Acknowledgements}

We thank J.E. Loth, J.R. Alexander, Y. Luo, K. Das and W. Shao for their contribution to this work. This work was supported by NIMH grants, by NIMH Research Scientist Award MH00176 (M.B.) and by the New York State Office of Mental Health. Correspondence to M.B.

Sir - Although a letter by our colleagues Bochetta et al. ${ }^{2}$ is entitled "Is bipolar disorder linked to Xq28?", no statistical evidence on linkage is presented. A Table is offered of G6PD deficiency in psychiatric patients in Sardinia, where males with bipolar disorder have $20.8 \%$ G6PD deficiency, and females have $36.4 \%$ (females are combined adding heterozygotes and homozygotes for deficiency). Comparisons of patients 